\section{Regards sur l'économie allemande}

Bulletin économique du CIRAC

$95 \mid 2010$

Varia

\title{
Arrêt de Karlsruhe à propos de Hartz IV : une loi conforme mais perfectible
}

Isabelle Bourgeois

\section{OpenEdition}

\section{Journals}

Édition électronique

URL : http://journals.openedition.org/rea/4034

DOI : 10.4000/rea.4034

ISBN : 978-2-8218-0886-7

ISSN : 1965-0787

Éditeur

CIRAC

\section{Édition imprimée}

Date de publication : 1 mars 2010

Pagination : 33-36

ISSN : 1156-8992

\section{Référence électronique}

Isabelle Bourgeois, «Arrêt de Karlsruhe à propos de Hartz IV : une loi conforme mais perfectible », Regards sur l'économie allemande [En ligne], 95 I mars 2010, mis en ligne le 01 juin 2010, consulté le 02 mai 2019. URL : http://journals.openedition.org/rea/4034 ; DOI : 10.4000/rea.4034 


\title{
Arrêt de Karlsruhe à propos de Hartz IV : une loi conforme mais perfectible
}

\author{
Isabelle Bourgeois
}

\begin{abstract}
"Ce n'est pas un tsunami, c'est juste une grosse vague Westerwelle [jeu de mot sur le nom du vice-chancelier FDP] ", ironise Horst Seehofer (CSU) dans son discours lors du "Mercredi des Cendres politique " de la CSU à Passau. Ces réunions qui closent traditionnellement la période du carnaval sont l'occasion pour les partis politiques de 'régler leurs comptes' au vitriol. Sigmar Gabriel (SPD) rappelle ainsi à Vilshofen que si, d'après le calendrier, « le règne des fous se termine aujourd'hui », il n'en va pas de même du gouvernement fédéral... Leur cible : Guido Westerwelle qui s'insurgeait, dans une tribune libre publiée sur Welt Online (11-02) contre la " tournure socialiste " prise par les débats qu'a déclenchés le jugement du Tribunal constitutionnel fédéral à propos de la Loi Hartz IV. "On débat de la question de savoir qui touchera plus ", au mépris de ces couches moyennes qui paient les impôts ; "promettre au peuple une prospérité sans effort est une invite au retour à l'ère de la Rome décadente ».

Le jugement, rendu le 9 février 2010, a déclenché un tir nourri de phrases assassines. Le climat politique est exacerbé à l'approche des élections du 9 mai en Rhénanie du Nord-Westphalie, enjeu crucial pour le FDP. Mais, plus important, l'arrêt, qui développe le concept du droit fondamental à un « minimum vital conforme à la dignité humaine ", a ravivé une plaie qui suppure depuis 2003 : la réforme d'un Etat providence dont l'hypertrophie est la principale cause de la dette. La Loi Hartz IV n'en est qu'une mesure, mais la plus douloureuse pour le SPD et la gauche extrême. Les esprits se sont échauffés au point que le président du Tribunal constitutionnel fédéral, Hans-Jürgen Papier, s'est vu contraint de faire une mise au point sur la portée du jugement : « il n'y est pas question d'une redéfinition de l'Etat social » (F.A.Z., 14-02). La Cour a simplement rappelé le législateur à son devoir de transparence et de réalisme dans le calcul des indemnités versées au titre de la Loi Hartz IV. Rude tâche, il est vrai, car il lui est ni plus ni moins demandé de dresser un catalogue chiffré des besoins concrets que doit assouvir la collectivité pour garantir le respect de la dignité humaine...
\end{abstract}

Les forfaits mensuels de base versés aux bénéficiaires du revenu de substitution prévu par la Loi Hartz IV ne sont pas contraires à la Constitution : «l'examen critique ne permet pas de les considérer comme insuffisants pour garantir un minimum vital conforme à la dignité humaine ", considère le Tribunal constitutionnel fédéral dans son arrêt du 9 février 2010 (BVerfG, 1 BvL 1/09, 1 BvL 3/09, 1 BvL 4/09). En revanche, leur mode de calcul est anticonstitutionnel, puisqu'il s'assimile ni plus ni moins à "une estimation 'à l'aveuglette' " ("ins Blaue hinein »). Les juges de Karlsruhe font donc injonction au législateur de définir des critères argumentés, transparents et tenant compte des besoins réels, notamment des enfants, pour calculer le montant du forfait de base. Les nouvelles dispositions doivent entrer en vigueur au 31 décembre 2010. Elles n'auront pas d'effet rétroactif.

La Loi Hartz IV ne fait pas, en tant que telle, l'objet du jugement. La Cour n'avait en effet été saisie par le Tribunal des affaires sociales du Land de Hesse et le Tribunal fédéral des affaires sociales que sur le point de savoir "si les dispositions du nouveau SGB II [chapitre II, révisé par la Loi Hartz IV, du Code social allemand: Sozialgesetzbuch] relatives au montant du forfait de base versé aux chômeurs
Calculer un forfait "à l'aveuglette " est contraire à la Constitution 
adultes, aux enfants de moins de 14 ans ou aux familles ayant des enfants de cet âge sont conformes à la Loi fondamentale » ou non (communiqué du 19-08-2009).

La Quatrième Loi pour des services modernes du marché de l'emploi : « Loi Hartz IV », adoptée le 24-12-2003 et entrée en vigueur le 01-01-2005, a simplifié le système d'indemnisation chômage, le réduisant à deux types de prestations :

- les indemnités chômage (Arbeitslosengeld I, ALG I), financées par les cotisations (principe d'assurance); leur montant est proportionnel au salaire antérieur du chômeur ;

-et les indemnités forfaitaires (Arbeitslosengeld II, ALG II), versées à l'expiration des droits à l'ALG I. Elles sont financées par l'impôt et relèvent du principe d'assistance.

Cet ALG /l est distinct de l'aide sociale (Sozialhilfe) dont ne bénéficient que les personnes se trouvant dans l'incapacité d'assurer leur subsistance par leurs propres forces. Le principal critère qui distingue ce groupe des deux catégories précédentes est la capacité de travail : au-delà de 3 heures quotidiennes, toute personne est considérée comme demandeur d'emploi et peut prétendre à l'ALG II.

L'ALG // est un 'revenu minimum d'insertion' (comparable au revenu de solidarité active en vigueur en France), comprenant un volet de (re)qualification/(ré)insertion et un soutien pécuniaire. Ce soutien prend la forme d'un forfait de base (Regelleistung) dont le montant était à l'origine fixé à $345 €$ à l'ouest et $331 €$ à l'est pour un célibataire avec ou sans enfant ; pour un couple, il est de $90 \%$ de cette somme par personne. A cela s'ajoutent, le cas échéant, des allocations logement et chauffage et des aides exceptionnelles. Le versement de ce soutien est fonction du degré de nécessité (Bedürftigkeit) du demandeur, car sont pris en considération dans le calcul de ce revenu de substitution de base toutes les sources de revenu comme le patrimoine du chômeur et de ses proches.

Peuvent bénéficier du forfait de base tous les demandeurs d'emploi de 15 à 65 ans. Les enfants vivant sous leur toit touchent un forfait dénommé Sozialgeld (indemnité sociale). Son montant est fonction de l'âge : $60 \%$ du forfait de base jusqu'à 14 ans révolus, puis $80 \%$. Dans le cadre des mesures de soutien à la consommation, une modification (valable du 01-07-2009 au 31-12-2011) a été apportée à ces dispositions : le groupe des moins de 15 ans est scindé en deux, et le taux porté à $70 \%$ pour les 7-14 ans.

En 2008, on comptait 6,9 millions de bénéficiaires de l'ALG // sous ses différentes formes, dont 1,9 million de célibataires, un peu plus de 650000 parents isolés et 1,8 million de moins de 15 ans (DIW-Wochenbericht, $n^{\circ} 6 / 2010$ ).

Un forfait de $345 €$ n'est pas anticonstitutionnel en soi

Un principe constitutionnel clef : la responsabilité individuelle
Contrairement à ce qu'ont pu laisser croire certains commentaires de l'arrêt, il ne s'agissait donc pas de déterminer si le montant de cette indemnité forfaitaire de base est constitutionnel ou non. Porté à $359 € \mathrm{le} 1^{\text {er }}$ juillet 2009 pour un adulte, il était de $345 €$ au moment de l'entrée en vigueur de la loi ; c'est ce dernier montant qui est au cœur de l'arrêt. Or c'est sur ces $345 €$ que se cristallisent outre-Rhin depuis l'origine les débats et les contentieux dont le pivot est le concept d'Etat social et la définition à donner à la notion de justice sociale qui le sous-tend : priorité à la responsabilité individuelle (équité des chances) ou au socialement souhaitable (égalité de traitement)?

Ainsi, le Tribunal fédéral des affaires sociales avait considéré, dans un arrêt fondamental rendu le 23 novembre 2006 (B 11b AS 1/06 R), que cette indemnité de $345 €$ remplit l'objectif fixé par la loi, à savoir assurer aux bénéficiaires un «minimum garanti » leur permettant de mener une "vie conforme à la dignité humaine »; elle leur confère en effet à la fois de quoi assurer leur subsistance («minimum vital») et participer à la vie sociale («minimum socio-culturel»). Instance de recours administratif suprême dans tout contentieux opposant les bénéficiaires de transferts sociaux à l'Etat, cette Cour avait tranché sur le principe, estimant qu'elle n'a " aucun doute fondé quant à la conformité avec la Constitution du montant des prestations réglementaires " (voir REA 79/06). Cet arrêt aussi avait suscité de très vives controverses. Et face à la multiplication des menaces de saisine du Tribunal constitutionnel fédéral sur la constitutionnalité de la loi Hartz IV, Hans-Jürgen Papier avait, déjà, été amené à souligner publiquement «que la Constitution n'interdit pas de réduire l'Etat social » et à rappeler que «les fondements de notre Constitution reposent sur le principe de la responsabilité individuelle » (Frankfurter Allgemeine Zeitung, 15-06-06).

\section{Le principe de l'aide à l'auto-assistance}

La loi Hartz IV cherchait à mettre fin à la dérive d'un Etat providence hypertrophié (aujourd'hui encore, les dépenses sociales accaparent près de la moitié du budget allemand). Elle l'a fait en renouant avec un principe fondateur de l'économie sociale de marché : l'aide à l'auto-assistance (Hilfe zur Selbsthilfe) qui est l'expression du principe de subsidiarité, autrement dit de cette responsabilité individuelle dont le corollaire est l'interdiction faite à l'Etat de se substituer à l'individu (principe de liberté).

Or au fil du temps, cette approche de la solidarité s'était diluée, donnant lieu à un « engrenage de répartition qui, en pénalisant toujours plus la performance et la prévoyance individuelles, entretient le développement d'une logique de revendication et d'une mentalité d'assisté ", ainsi que le formulait en 1982 déjà Otto Graf Lambsdorff, ministre des Finances (FDP) du chancelier Schmidt (SPD). Ce n'est qu'en 2003 que le chancelier Schröder (SPD) rompra cet engrenage avec les réformes sociales de l'Agenda 2010.

Ce principe est le pivot de la Loi Hartz IV
Or c'est ce concept de responsabilité individuelle qui est au cœur des débats sur l'Etat social et son « démantèlement »- ou non, selon les perspectives -, par la loi Hartz IV. Celle-ci avait été placée expressément sous le signe du «Fördern und 
Fordern » : la collectivité a le devoir de soutenir les personnes en difficulté et, en retour, elle est en droit d'exiger que celles-ci fassent l'effort de se réinsérer. C'est ainsi que la loi Hartz IV $(\S 1,1)$ vise à « renforcer la responsabilité individuelle des personnes nécessiteuses à capacité de travail entière, comme de ceux qui vivent avec elles dans une communauté de besoins, et à contribuer à ce qu'elles soient capables d'assurer leur subsistance indépendamment du minimum garanti, c'est-àdire par leurs propres forces et moyens ". C'est cette approche qui a présidé au choix d'un forfait de base de $345 €$ : il s'agit d'un soutien passager accordé à une personne en quête active d'un emploi ; son montant doit donc être nettement inférieur à un bas salaire afin d'inciter au retour en emploi.

Les résistances à ce rééquilibrage entre droits et devoirs respectifs de l'individu et de la collectivité ont été très vives, amenant $G$. Schröder à démissionner de la tête d'un SPD profondément divisé et à provoquer des élections anticipées au Bundestag en 2005. Depuis, la gauche du SPD, Die Linke, de même qu'une frange des Verts allemands n'ont de cesse de forcer un retour en arrière sur la réforme. Or leur quête de plus de justice sociale, déclinée également via la revendication d'un salaire minimum légal (voir l'analyse de K. Brenke dans REA 94/09) se base sur le principe du socialement souhaitable et de la lutte contre la pauvreté. Le montant du revenu de substitution (ou salaire) doit être fonction des besoins de l'individu qui les perçoit et non pas de considérations macro-économiques. A l'évidence, une telle approche est susceptible de rencontrer un large écho dans l'opinion - c'est cette «tournure socialiste » des débats que pointe G. Westerwelle, s'attirant les critiques tous azimuts. Car la loi Hartz IV a laissé un malaise jusque dans les rangs de la CSU et de l'aile sociale de la CDU - ravivé en période de campagne électorale, quand les considérations de politique sociale l'emportent sur celles de politique économique et budgétaire. Personne n'ose alors rompre ouvertement avec « l'utopie de l'Etat social ", remarque le constitutionnaliste CDU Rupert Scholz (Welt Online, 18-02-2010). L'Etat Providence ne peut pourtant distribuer les richesses que si son financement est durable...

La Cour de Karlsruhe en est consciente: les modifications n'auront pas d'effet rétroactif pour ne pas nuire à la santé des finances publiques. Car selon la jurisprudence, le législateur n'est en effet pas tenu de réviser rétroactivement une disposition incompatible avec la Loi fondamentale notamment "lorsque cela va à l'encontre d'une programmation budgétaire régulière » (point 217). II n'est pas tenu non plus d'augmenter le forfait de base : "comme rien ne permet de conclure à l'évidence que [celui-ci] est insuffisant, le législateur n'est pas tenu directement par la Constitution de fixer des montants supérieurs. II doit plutôt mettre à exécution une procédure pour déterminer, conformément à la réalité et aux besoins, et dans le respect des considérations d'ordre constitutionnel..., les prestations nécessaires à la garantie d'un minimum vital conforme à la dignité humaine et ancrer dans la loi un droit à ces prestations » (point 211). L'arrêt précise : « en raison du pouvoir discrétionnaire du législateur..., le Tribunal constitutionnel fédéral n'est pas habilité à agir pour fixer le montant des prestations » (point 212).
Un choix cornélien entre le socialement souhaitable et l'avenir durable de l'Etat Providence
Les juges de Karlsruhe ne demandent pas un forfait supérieur...

\section{Comment est fixé le montant du forfait de référence de $345 €$ pour un célibataire}

Le modèle statistique prend pour base les sondages sur les revenus et la consommation (EVS) menés tous les 5 ans par Destatis. Pour déterminer le forfait de référence, ne sont considérés que les $20 \%$ des ménages à une personne aux revenus nets les plus bas, hors bénéficiaires de l'aide sociale. L'estimation des besoins des « récipiendaires de Hartz IV » se fait alors au prorata des dépenses de ces ménages. Ainsi, par exemple, ne sont pris en considération que $96 \%$ des postes " alimentation, boissons et tabac », $89 \%$ de " vêtements et chaussures », $64 \%$ des dépenses de santé, $42 \%$ pour "loisirs, divertissement et culture » ou $37 \%$ pour les transports. Au $1^{\text {er }}$ janvier 2007 , cette évaluation, qui reposait sur le sondage EVS de 1998, a été revue en fonction de la vague 2003 (par exemple, le taux du poste " courrier » est passé de $64 \%$ à $75 \%$ ). Et elle a changé de base : la référence est désormais l'évolution des salaires bruts et cotisations, telle qu'elle s'applique au calcul des pensions retraite.

Ce qui rend inconstitutionnelle la méthode selon laquelle le législateur a fixé le forfait de base, c'est qu'elle est arbitraire. Le modèle statistique empirique n'est pas incriminé, puisqu'il reflète la réalité à un instant $T$. Par contre la manière dont est ensuite évalué le minimum vital n'est ni transparente ni adaptée aux besoins réels du bénéficiaire. Elle est contraire à la Constitution parce que le législateur "s'est écarté sans justification objective des principes structurants du modèle statistique
... mais le législateur doit justifier ses choix 
II doit prendre en charge les besoins exceptionnels de certains bénéficiaires

Priorité de politique sociale : définir les besoins propres des enfants scolarisés qu'il avait lui-même choisi » (point 173). II a évalué "à l'aveuglette " les besoins des bénéficiaires. Pour quelle raison a-t-on changé de référence pour les calculs en 2007 ? Et pourquoi par exemple les dépenses de formation/éducation ne sont-elles pas prises en compte ? Certes, le gouvernement fédéral argue de la souveraineté des Länder en matière de formation, mais les Länder ont à en financer les infrastructures ; la politique sociale relève, elle, des prérogatives du Bund.

Par ailleurs, l'aide étant forfaitaire, les besoins exceptionnels sont insuffisamment pris en considération, dès lors qu'ils sont inscrits dans la durée ; l'aide ponctuelle sous la forme d'un crédit remboursable, telle qu'elle s'applique au renouvellement d'une machine à laver ou d'une paire de lunettes, est en effet inadaptée. Ce point est le plus simple à corriger pour le législateur. Dès le 17 février, le ministère fédéral du Travail et des Affaires sociales a présenté une première liste de besoins exceptionnels définis conjointement avec l'Agence fédérale pour l'emploi (médicaments non remboursés dans le cas de certaines maladies chroniques, frais de transport et d'hébergement pour les enfants de couples divorcés, aide ménagère pour un malade en fauteuil roulant...). Ils sont couverts dès à présent.

"Le législateur n'a pas évalué le minimum vital d'un enfant mineur». Or «les enfants ne sont pas des adultes miniatures"; l'évaluation de leurs besoins "doit prendre en considération les phases dans l'évolution de l'enfance et ce qui est nécessaire au développement de la personnalité d'un enfant " (point 191). En ce qui concerne les écoliers, les juges se font très précis: "Les dépenses nécessaires pour qu'ils puissent remplir leurs devoirs vis-à-vis de l'Ecole font partie de leurs besoins existentiels. Si ces besoins ne sont pas couverts, les enfants nécessiteux risquent l'exclusion des chances que leur offre la vie ". Sans " manuels scolaires, cahiers ou calculatrices ", ils courent en effet le "danger de ne pas être en mesure, plus tard, de subvenir à leurs besoins par leurs propres forces » Or cela est incompatible avec les deux principes constitutionnels au cœur de l'arrêt: la "dignité de l'être humain ", que les " pouvoirs publics ont l'obligation de respecter et de protéger " (art. 1, al. 2 de la Loi fondamentale), en liaison avec le principe d'« Etat fédéral démocratique et social » (art. 20, al. 1) de la RFA.

EN EXIGEANT UN SOUTIEN CORRESPONDANT AUX BESOINS, le Tribunal constitutionnel fédéral enjoint au législateur de mieux respecter le principe d'équité des chances au fondement de l'Etat social allemand. Mais comment concilier au concret cette approche forcément centrée sur l'individu avec celle, par nature générale, de l'Etat Providence ? Cette question, qui relève de la philosophie du droit, doit trouver une réponse dans un autre registre, celui de la loi. C'est là qu'est la portée générale de cet arrêt. Le fait que le législateur ne dispose pour ce faire que de quelques mois, en pleine période électorale de surcroît, n'est pas propice à une réflexion sereine, intégrant l'ensemble des aspects à considérer à la fois sous l'angle de la politique économique, budgétaire et sociale. Le climat s'y prête d'autant moins qu'une récente étude de l'OCDE sur les chômeurs de longue durée a remis en tête de l'agenda le thème de la pauvreté, un thème dont l'UE compte faire sa nouvelle priorité lors du sommet de printemps. Cela ouvre la voie à la dramatisation et à l'amalgame dans une société en proie à la "phobie de la précarité ", comme le formule R. Nahrendorf, ancien rédacteur en chef du quotidien Handelsblatt (ibid., 15-02). Or ce que demandent les juges en se focalisant sur les écoliers et leurs besoins en matière de formation, c'est le retour à un modèle de société où le travail et le mérite individuel activent l'ascenseur social, et donc fondé sur le respect de ces couches moyennes performantes au « milieu » de la société allemande et de sa prospérité. Pour réduire les disparités sociales, il ne suffit pas de couvrir les besoins physiques des « bénéficiaires de Hartz IV » et de leur permettre la participation à la vie culturelle et sociale - il faut avant tout favoriser et développer leur accès à la formation.

\section{Indications bibliographiques}

- BourgeoIs I. (dir.), Le modèle social allemand en mutation, Travaux et Documents du CIRAC, Cergy-Pontoise, 2005

- http://www.bundesverfassungsgericht.de/entscheidungen/ls20100209_1bvl000109.html

- http://www.bundesverfassungsgericht.de/pressemitteilungen/bvg10-005.html

- http://www.bundesverfassungsgericht.de/en/press/bvg10-005en.html

- http://www.bundesverfassungsgericht.de/pressemitteilungen/bvg09-096.html 